\title{
Formal Languages - Concatenation and Closure
}

\author{
Michał Trybulec \\ Motorola Software Group \\ Cracow, Poland
}

\begin{abstract}
Summary. Formal languages are introduced as subsets of the set of all 0 -based finite sequences over a given set (the alphabet). Concatenation, the $n$-th power and closure are defined and their properties are shown. Finally, it is shown that the closure of the alphabet (understood here as the language of words of length 1) equals to the set of all words over that alphabet, and that the alphabet is the minimal set with this property. Notation and terminology were taken from [5] and [13].
\end{abstract}

MML identifier: FLANG_1, version: 7.8 .04 4.81.962

The terminology and notation used here are introduced in the following articles: [10], [4], [11], [8], [9], [2], [14], [3], [1], [6], [12], and [7].

\section{Preliminaries}

For simplicity, we follow the rules: $E$ is a set, $x$ is a set, $A, B, C, D$ are subsets of $E^{\omega}, a, b, c$ are elements of $E^{\omega}, e$ is an element of $E, i, n, n_{1}, n_{2}, m$ are natural numbers, and $p, q, r_{1}, r_{2}$ are real numbers.

Let us consider $E, a, b$. Then $a^{\frown} b$ is an element of $E^{\omega}$.

Let us consider $E$. Then \langle\rangle$_{E}$ is an element of $E^{\omega}$.

Let $E$ be a non empty set and let $e$ be an element of $E$. Then $\langle e\rangle$ is an element of $E^{\omega}$.

Let us consider $E, a$. Then $\{a\}$ is a subset of $E^{\omega}$.

Let us consider $E$, let $f$ be a function from $\mathbb{N}$ into $2^{E^{\omega}}$, and let us consider $n$. Then $f(n)$ is a subset of $E^{\omega}$.

One can prove the following propositions: 
(1) If $\{x\} \not \subset X$, then $\{x\}$ misses $X$.

(2) If $n_{1}>1$ or $n_{2}>1$, then $n_{1}+n_{2}>1$.

(3) $n>0$ iff $n \geq 1$.

(4) If $r_{1}+p \leq r_{2}+q$ and $p \geq q$, then $r_{1} \leq r_{2}$.

(5) If $n_{1}+n \leq n_{2}+1$ and $n>0$, then $n_{1} \leq n_{2}$.

(6) $n_{1}+n_{2}=1$ iff $n_{1}=1$ and $n_{2}=0$ or $n_{1}=0$ and $n_{2}=1$.

(7) $\quad a^{\frown} b=\langle x\rangle$ iff $a=\langle\rangle_{E}$ and $b=\langle x\rangle$ or $b=\langle\rangle_{E}$ and $a=\langle x\rangle$.

(8) For all finite 0 -sequences $p, q$ such that $a=p^{\frown} q$ holds $p$ is an element of $E^{\omega}$ and $q$ is an element of $E^{\omega}$.

(9) If $\langle x\rangle$ is an element of $E^{\omega}$, then $x \in E$.

(10) If len $b=n+1$, then there exist $c, e$ such that len $c=n$ and $b=c^{\frown}\langle e\rangle$.

(11) If $a^{\frown} a=a$, then $a=\emptyset$.

\section{Concatenation of Languages}

Let us consider $E, A, B$. The functor $A \frown B$ yields a subset of $E^{\omega}$ and is defined by:

(Def. 1) $x \in A \frown B$ iff there exist $a, b$ such that $a \in A$ and $b \in B$ and $x=a \frown b$.

The following propositions are true:

(12) $A \frown B=\emptyset$ iff $A=\emptyset$ or $B=\emptyset$.

(13) $A \frown\left\{\langle\rangle_{E}\right\}=A$ and $\left\{\langle\rangle_{E}\right\} \frown A=A$.

(14) $A \frown B=\left\{\langle\rangle_{E}\right\}$ iff $A=\left\{\langle\rangle_{E}\right\}$ and $B=\left\{\langle\rangle_{E}\right\}$.

(15) \langle\rangle$_{E} \in A \frown B$ iff \langle\rangle$_{E} \in A$ and \langle\rangle$_{E} \in B$.

(16) If \langle\rangle$_{E} \in B$, then $A \subseteq A \frown B$ and $A \subseteq B \frown A$.

(17) If $A \subseteq C$ and $B \subseteq D$, then $A \frown B \subseteq C \frown D$.

(18) $(A \frown B) \frown C=A \frown(B \frown C)$.

(19) $A \frown(B \cap C) \subseteq(A \frown B) \cap(A \frown C)$ and $(B \cap C) \frown A \subseteq(B \frown A) \cap(C \frown A)$.

(20) $A \frown B \cup A \frown C=A \frown(B \cup C)$ and $B \frown A \cup C \frown A=(B \cup C) \frown A$.

(21) $A \frown B \backslash A \frown C \subseteq A \frown(B \backslash C)$ and $B \frown A \backslash C \frown A \subseteq(B \backslash C) \frown A$.

(22) $A \frown B \cdot A \frown C \subseteq A \frown(B \dot{-} C)$ and $B \frown A \cdot C \frown A \subseteq(B \cdot C) \frown A$.

\section{3. $n$-TH POWer of A Language}

Let us consider $E, A, n$. The functor $A^{n}$ yields a subset of $E^{\omega}$ and is defined by:

(Def. 2) There exists a function $c_{1}$ from $\mathbb{N}$ into $2^{E^{\omega}}$ such that $A^{n}=c_{1}(n)$ and $c_{1}(0)=\left\{\langle\rangle_{E}\right\}$ and for every $i$ holds $c_{1}(i+1)=c_{1}(i) \frown A$. 
Next we state a number of propositions:

(23) $\quad A^{n+1}=\left(A^{n}\right)^{\frown} A$.

(24) $A^{0}=\left\{\langle\rangle_{E}\right\}$.

(25) $A^{1}=A$.

(26) $A^{2}=A \frown A$.

(27) If $n \geq 1$, then $\left(\emptyset_{E^{\omega}}\right)^{n}=\emptyset$.

(28) $\left\{\langle\rangle_{E}\right\}^{n}=\left\{\langle\rangle_{E}\right\}$.

(29) $A^{n}=\left\{\langle\rangle_{E}\right\}$ iff $n=0$ or $A=\left\{\langle\rangle_{E}\right\}$.

(30) If \langle\rangle$_{E} \in A$, then \langle\rangle$_{E} \in A^{n}$.

(31) $\left(A^{n}\right)^{\frown} A=A \frown A^{n}$.

(32) $\quad A^{m+n}=\left(A^{m}\right) \frown A^{n}$.

(33) $\left(A^{m}\right)^{n}=A^{m \cdot n}$.

(34) If \langle\rangle$_{E} \in A$ and $n>0$, then $A \subseteq A^{n}$.

(35) If \langle\rangle$_{E} \in A$ and $n>0$ and $m>n$, then $A^{n} \subseteq A^{m}$.

(36) If $A \subseteq B$, then $A^{n} \subseteq B^{n}$.

(37) $A^{n} \cup B^{n} \subseteq(A \cup B)^{n}$.

(38) $(A \cap B)^{n} \subseteq A^{n} \cap B^{n}$.

(39) If $a \in C^{m}$ and $b \in C^{n}$, then $a^{\frown} b \in C^{m+n}$.

\section{Closure of a Language}

Let us consider $E, A$. The functor $A^{*}$ yielding a subset of $E^{\omega}$ is defined as follows:

(Def. 3) $A^{*}=\bigcup\left\{B: \bigvee_{n} B=A^{n}\right\}$.

The following propositions are true:

(40) $x \in A^{*}$ iff there exists $n$ such that $x \in A^{n}$.

(41) $A^{n} \subseteq A^{*}$.

(42) If $x \in A$, then $x \in A^{*}$.

(43) $A \subseteq A^{*}$.

(44) $A \frown A \subseteq A^{*}$.

(45) If $a \in C^{*}$ and $b \in C^{*}$, then $a^{\frown} b \in C^{*}$.

(46) If $A \subseteq C^{*}$ and $B \subseteq C^{*}$, then $A \frown B \subseteq C^{*}$.

(47) $A^{*}=\left\{\langle\rangle_{E}\right\}$ iff $A=\emptyset$ or $A=\left\{\langle\rangle_{E}\right\}$.

(48) \langle\rangle$_{E} \in A^{*}$.

(49) If $A^{*}=\{x\}$, then $x=\langle\rangle_{E}$.

(50) If $x \in A^{m+1}$, then $x \in\left(A^{*}\right)^{\frown} A$ and $x \in A \frown A^{*}$.

(51) If $x \in\left(A^{*}\right)^{\frown} A$ or $x \in A \frown A^{*}$, then $x \in A^{*}$. 
(52) If \langle\rangle$_{E} \in A$, then $A^{*}=\left(A^{*}\right)^{\frown} A$ and $A^{*}=A \frown A^{*}$.

(53) If \langle\rangle$_{E} \in A$, then $A^{*}=\left(A^{*}\right) \frown A^{n}$ and $A^{*}=\left(A^{n}\right) \frown A^{*}$.

(54) $A^{*}=\left\{\langle\rangle_{E}\right\} \cup A \frown A^{*}$ and $A^{*}=\left\{\langle\rangle_{E}\right\} \cup\left(A^{*}\right) \frown A$.

(55) $\quad A \frown A^{*}=\left(A^{*}\right) \frown A$.

(56) $\left(A^{n}\right) \frown A^{*}=\left(A^{*}\right) \frown A^{n}$.

(57) If $A \subseteq B^{*}$, then $A^{n} \subseteq B^{*}$.

(58) If $A \subseteq B^{*}$, then $A^{*} \subseteq B^{*}$.

(59) If $A \subseteq B$, then $A^{*} \subseteq B^{*}$.

(60) $\left(A^{*}\right)^{*}=A^{*}$.

(61) $\left(A^{*}\right) \frown A^{*}=A^{*}$.

(62) $\left(A^{n}\right)^{*} \subseteq A^{*}$.

(63) $\left(A^{*}\right)^{n} \subseteq A^{*}$.

(64) If $n>0$, then $\left(A^{*}\right)^{n}=A^{*}$.

(65) If $A \subseteq B^{*}$, then $B^{*}=(B \cup A)^{*}$.

(66) If $a \in A^{*}$, then $A^{*}=(A \cup\{a\})^{*}$.

(67) $A^{*}=\left(A \backslash\left\{\langle\rangle_{E}\right\}\right)^{*}$.

(68) $A^{*} \cup B^{*} \subseteq(A \cup B)^{*}$.

(69) $(A \cap B)^{*} \subseteq A^{*} \cap B^{*}$.

(70) $\langle x\rangle \in A^{*}$ iff $\langle x\rangle \in A$.

\section{Alphabet as a Language}

Let us consider $E$. The functor Lex $E$ yielding a subset of $E^{\omega}$ is defined by: (Def. 4) $x \in \operatorname{Lex} E$ iff there exists $e$ such that $e \in E$ and $x=\langle e\rangle$.

Next we state three propositions:

(71) $a \in(\operatorname{Lex} E)^{\operatorname{len} a}$.

(72) $(\operatorname{Lex} E)^{*}=E^{\omega}$.

(73) If $A^{*}=E^{\omega}$, then Lex $E \subseteq A$.

\section{REFERENCES}

[1] Grzegorz Bancerek. The ordinal numbers. Formalized Mathematics, 1(1):91-96, 1990.

[2] Czesław Byliński. Functions and their basic properties. Formalized Mathematics, 1(1):5565, 1990.

[3] Czesław Byliński. Functions from a set to a set. Formalized Mathematics, 1(1):153-164, 1990.

[4] Czesław Byliński. Some basic properties of sets. Formalized Mathematics, 1(1):47-53, 1990.

[5] John E. Hopcroft and Jeffrey D. Ullman. Introduction to Automata Theory, Languages and Computation. Addison-Wesley Publishing Company, 1979.

[6] Beata Padlewska. Families of sets. Formalized Mathematics, 1(1):147-152, 1990.

[7] Karol Pạk. The Catalan numbers. Part II. Formalized Mathematics, 14(4):153-159, 2006. 
[8] Andrzej Trybulec. Subsets of complex numbers. To appear in Formalized Mathematics.

[9] Andrzej Trybulec. Domains and their Cartesian products. Formalized Mathematics, 1(1):115-122, 1990.

[10] Andrzej Trybulec. Tarski Grothendieck set theory. Formalized Mathematics, 1(1):9-11, 1990.

11] Zinaida Trybulec. Properties of subsets. Formalized Mathematics, 1(1):67-71, 1990.

[12] Tetsuya Tsunetou, Grzegorz Bancerek, and Yatsuka Nakamura. Zero-based finite sequences. Formalized Mathematics, 9(4):825-829, 2001.

[13] William M. Waite and Gerhard Goos. Compiler Construction. Springer-Verlag New York Inc., 1984.

[14] Edmund Woronowicz. Relations defined on sets. Formalized Mathematics, 1(1):181-186, 1990.

Received March 9, 2007 